\title{
THE DILEMMA OF THE UNUSUAL DISTRIBUTION OF MR ENCEPHALOPATHY IN PRE-ECLAMPTIC/ECLAMPTIC FEMALE PATIENTS : DETECTION AND PITFALLS
}

\author{
Ghada Abdulmonaem $^{a}{ }_{*}$, RaniaAl-molla ${ }^{a, 1}$, Hosam N. Almassry ${ }^{a, 2}$, Hala Mwafi ${ }^{b, 3}$ \\ aRadiodiagenesis Deprtment, Faculty of Medicine, Zagazig University, Sharkia, Egypt. \\ b Obstetric\& Gynecology Department, Faculty of Medicine, Zagazig University, Sharkia, Egypt.
}

\begin{abstract}
Purposes: To evaluate the MRI findings in pre-eclamptic/ eclamptic encephalopathy and to evaluate the usefulness of diffusion weighted MRI (DW-MRI) as an imaging tool and its prognostic implication in changing treatment protocol. Patients \& methods: Prospective study included24 patients, diagnosed clinically as preeclampsia (14 cases) and eclampsia in (10 cases). Their ages ranged from 25 to 45 years with mean age of 35.6 years. They examined on a standard 1.5 Tesla MR unit. Initially conventional T2 and FLAIRimages were done followed by Diffusion weighted imaging. Results: All 24 patients were acutely hypertensive at presentation. All patients develop abnormal increased signal on T2 and FLAIR sequences predominantly involving the posterior circulation. 20 ofthese patients demonstrate increased SI on diffusion weighted images denoting vasogenic edema while only 4 patients showed corresponding low signal on DW-MRI (cytotoxic edema). Follow up study confirmed the resolution of the lesions (in 20 patients) successfully with reversible neurologic impairment while those 4 patients with cytotoxic edema pattern,they develop permanent T2 / FLAIR hyper intense signal and neurological impairment. Conclusion: Involvement of the parietal and occipital lobes is common in patients with eclampsia, and the signal abnormalities on MRI are reversible if recognized and treated early.DWI can correlates well with the patient's outcome, and should be performed in all eclamptic patients and that may affect greatly their management.
\end{abstract}

\section{INTRODUCTION}

$\mathbf{P}$ reeclampsia (PE) is a pregnancy-specific disease which, in addition to other hypertensive disorders, is an important cause of maternal and perinatal morbidity and mortality. Preeclampsia is diagnosed clinically by development of hypertension, protinurea, and edema after the 20th week of gestation which progress to eclampsia if seizures developed before, during or after delivery(1). The most common clinical presentation is known as posterior reversible encephalopathy syndrome (PRES) which is characterized by rapidly progressive signs and symptoms including headache, seizures, visual changes and occasionally new-onset seizures if encephalopathy developed (2).

The difference between hypertensive encephalopathy and PRES is that PRES can develop without a significant elevation in blood pressure (3)

Detecting the cause and pathogenesis is of great importance in directing therapy. If spasm induced thrombosis \& ischemia is the cause, so cytotoxic edema is responsible for the radiological findings and a clinician might tend to decrease intracranial pressure to maintain cerebral perfusion or to prescribe anticoagulant to prevent ischemic events. However if breakthrough of auto regulation is the cause, reduction in blood pressure alone could be the appropriate management while anticoagulation mightresult in hemorrhage $(4,5,6,7)$.Posterior Reversible Encephalopathy Syndrome (PRES) is a diverse clinicoradiologic syndrome with diverse clinical presentations and characteristic MRI features (8).

Symmetrically distributed areas of T2 hyperinensity predominantly within the territories of the posterior circulation is the typical MR imaging abnormalities. These radiological findings are usually but invariably resolve after normalization of blood pressure although may progress to ischemia and infarction if unrecognized or intracerbral hemorrhage may occur in whom hypertension cannot be controlled(9). 
Echo-planar diffusion-weighted MR imaging (DW-MRI) is a technique by which images sensitive chiefly to the diffusion of water molecules can be generated. DWI has the reliability to distinguish vasogenic edema which seen in PRES from cytotoxic edema in the setting of cerebral ischemia(10).

Aim of the work is to evaluate the MRI findings of pre-eclamptic/eclamptic encephalopathy and to evaluate the clinical usefulness of diffusion weighted MR imaging as a diagnostic tool and its prognostic implication in changing the patient's treatment protocol by determining the cause of edema in hypertensive encephalopathy (cytotoxic versus vasogenic).

\section{PATIENTS \& METHODS}

2.1. Selection of patients:

This prospective study included 24 patients (ages ranged from 25 to 45 years with mean age of 35.6 years) presented with signs and symptoms of pre-eclamptic/eclamptic encephalopathy All patients results were reviewed over a period of 17 months from April 2013 to august 14.The study was conducted in Radiodiagnosis Department-Zagazig University from Oby/Gynand/or Neurological Departmenst.

The patients were subjected to the following:

\subsubsection{Clinical examination:}

- All patients were examined by obstetrician and neurologist immediately after the clinical presentation and daily until signs subside.

- Informed consent obtained from patients or from their relatives

- Through physical examination was performed including blood pressure measurement during the course of the disease. Patients with eclampsia are usually monitored in an intensive care setting, so activity is limited and close observation of the blood pressure of pregnant women is very important. In general, the diagnosis of hypertension was defined when the blood pressure is
140/90 mmHg or greater after 20 weeks gestation in a previously normotensive woman and returns to normal less than 12 weeks postpartum according to the protocol established by the working group on high blood pressure in pregnancy and the American college of obstetricians and gynecology(11). Some authors accept a rise of $15 \mathrm{~mm} \mathrm{Hg}$ in diastolic and $30 \mathrm{~mm} \mathrm{Hg}$ in systolic blood pressure from the baseline as diagnostic of pregnancy induced hypertension(12).

- Laboratory studies showed hemoconcentration\& proteinuria (more than $300 \mathrm{mg} / 24 \mathrm{~h}$ collection of urine) in all patients.

- Treatment consists of antihypertensive drugs in all patients, antiepileptic drugs and supplementary magnesium sulphate in all the patients who experience seizures.

2.1.2. Inclusion criteria:

- All patients with acutely raised blood pressure for several hours or days before MR examination.

- Patients presented with headache, seizures, and/or visual disturbances examined by MRI within 1 day of the onset of signs or symptoms.

- Patients presenting by a frank seizures attack, the examinations were done within 3 days period after onset of symptoms

2.1.3. Exclusion criteria:

- Past history of hypertension or renal diseases.

- Past history of cortical and subcortical white matter lesions.

- Eclamptic patients in the ictal and immediately post ictal state (restricted diffusion seen in the ictal and immediately post ictal state).

- Patient with absolute or relative contraindication for MR examination.

2.2. MRI Technique: 
2.2.1Conventional magnetic resonance imaging (MRI):

All MRI studies were done using Philips machine (01.5 Tesla). All patients were asked to get rid of any metallic subjects as well as they were asked about any contraindication to MRI examination (artificial heart valve, cardiac pacemaker, metallic stents or joint prosthesis except that made of titanium). The patients were informed about the duration of the examination, the position of the patient and the importance of being motionless.

MRI study was done with the patients in the supine position using the standard head coil. The examination was done before contrast administration, a scout sagittal T1-weighted view was obtained to verify the precise position of the patient and to act as a localizer for subsequent slices, then multiple pulse sequences were used to obtain axial images followed by coronal and/or sagittal images based on the location of the pathology encountered. In midline lesions sagittal planes were used while in laterally located lesions coronal images were more helpful.

2.2.2.Diffusion weighted MR imaging (DWI)

The imaging sequence for DWI was a multi-section single shot spin echo EPI sequence (TR/TE/NEX: 4200/140 ms/I) with

A

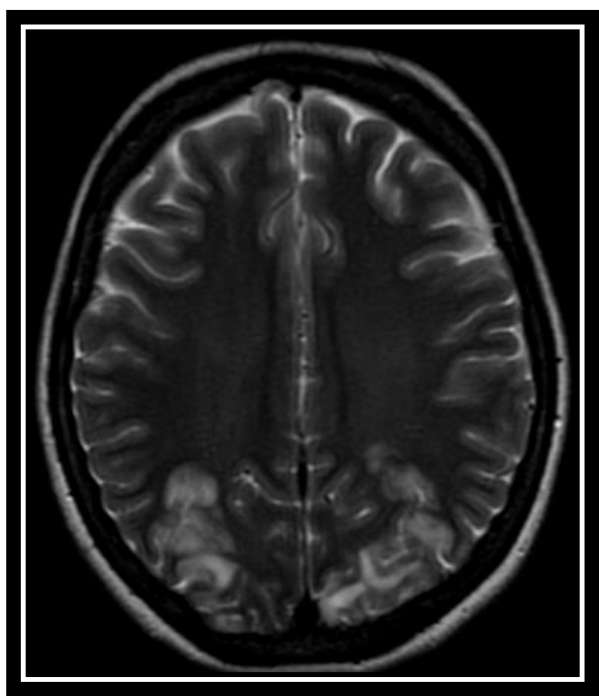

diffusion sensitivities

of

b

values $=1000 \mathrm{~s} / \mathrm{mm} 2$. The diffusion gradients were applied sequentially in three orthogonal directions ( $\mathrm{X}, \mathrm{Y} \& \mathrm{Z}$ directions). Sections of $5 \mathrm{~mm}$ thickness, interslice gap of $1 \mathrm{~mm}$, FOV $240 \mathrm{~mm}$ and a matrix of $128 \times 256$ were used for all images. The total acquisition time was $80 \mathrm{~s}$.

2.3. Image analysis

In all patients, MRIs were examined looking for abnormal hyperintense lesions on T2 weighted \& FLAIR images. Site of affection were noted either posterior or frontal white matter. The areas of abnormal signal intensity on FLAIR or conventional T2 were compared with areas of abnormalities seen on the corresponding diffusion weighted images and ADC maps. ADC was generated from DW images, which serve as reference to decide whether corresponding lesions present on $\mathrm{T} 2$ weighted images, had increased diffusuibilty or not. Follow up MR showed disappearance of all previously seen $\mathrm{T} 2$ hyperinensity in 20 patients with total clinical improvement, the remaining four patients, showed persistent signal abnormality with persistent neurological deficit in the results.

B

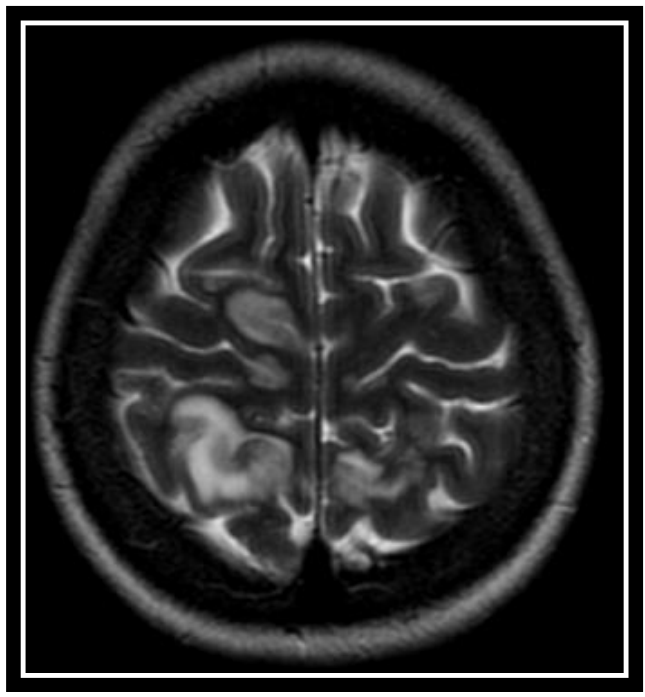


$\mathrm{C}$

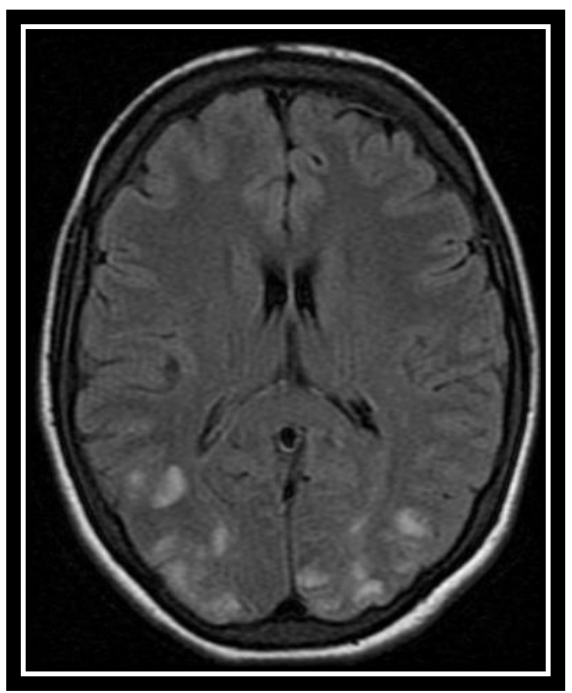

$\mathrm{E}$

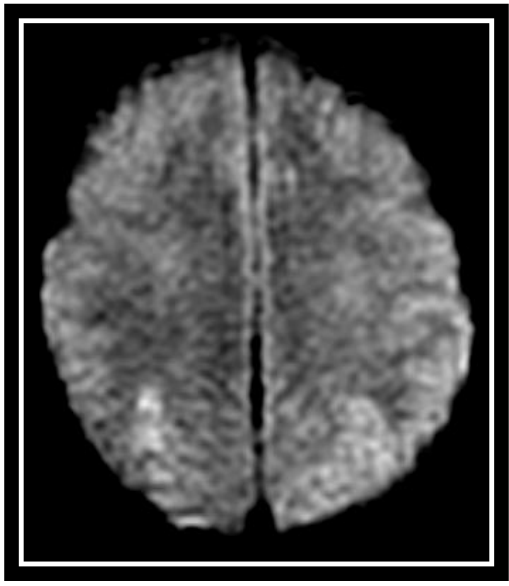

G

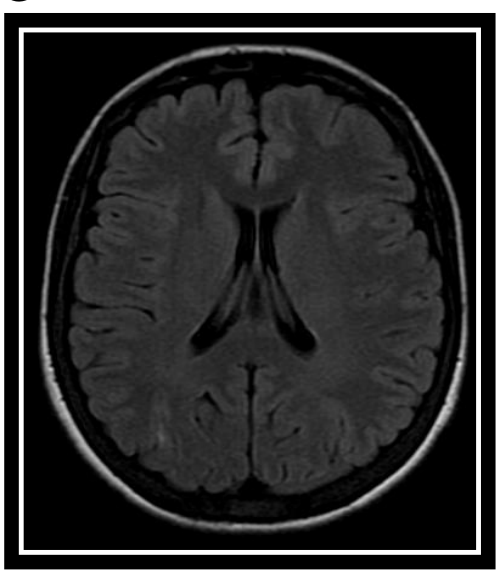

$\mathrm{D}$

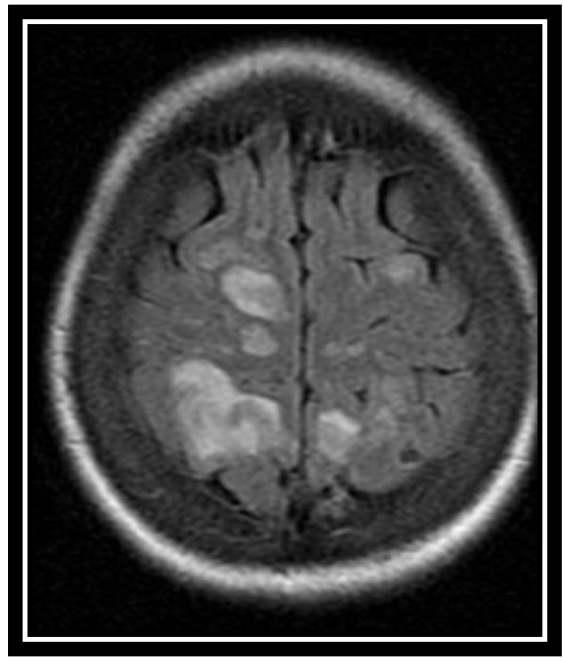

$\mathrm{F}$

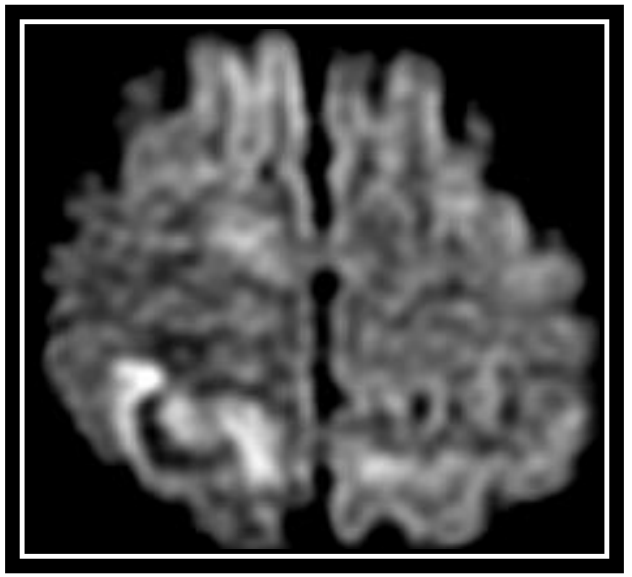

Fig. 1 (a-g): 31 - year-old woman (patient no. 2 in table 1) with blurred vision and disturbed conscious level due to eclampsia. Fig.(a\&b): Axial T2 WIs and (c,d) axial FLAIR Images show bilateral almost symmetrical cortical and sub cortical hyperintense patches in the parieto-occipital lobes as ell as both frontal lobes. Fig.(e,f): DWI showed small areas not corresponding to the abnormalities on T2 WIs and FLAIR displaying high signal on diffusion images and high signal on ADC map (suggestive of vasogenic edema and T2 shin through). Fig.(g):Follow-up FLAIR image: shows almost complete resolution of the previously described abnormal high T2 and FLAIR signal intensity (confirming vasogenic edema). 

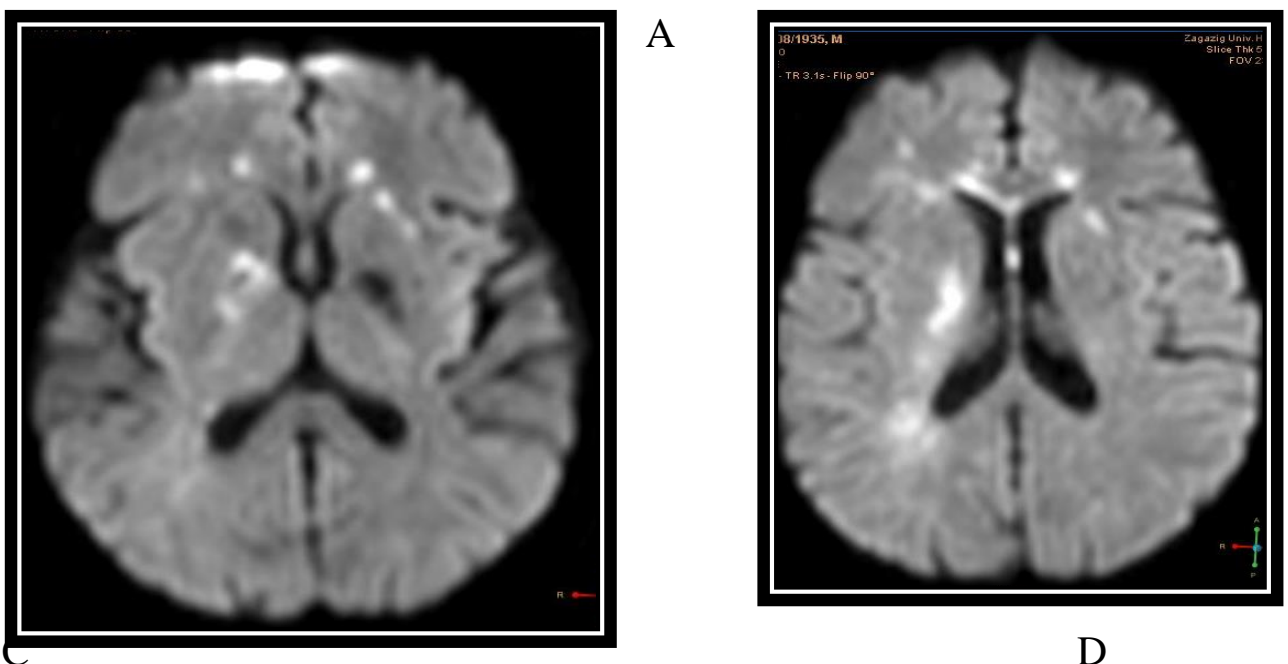

B

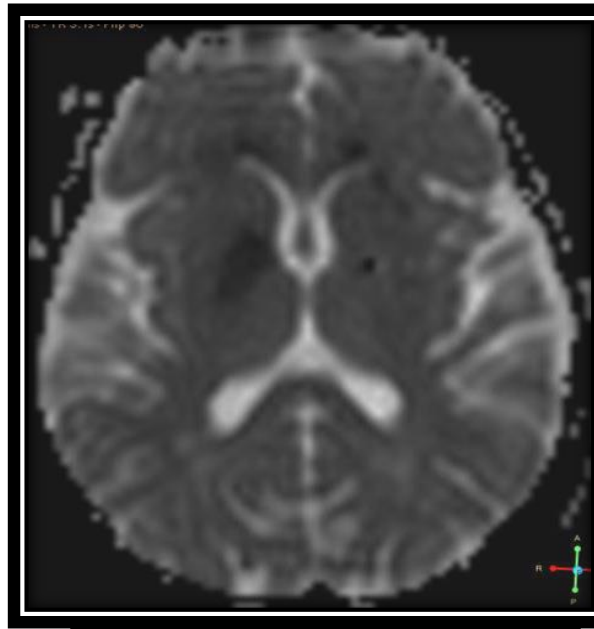

$\mathrm{D}$
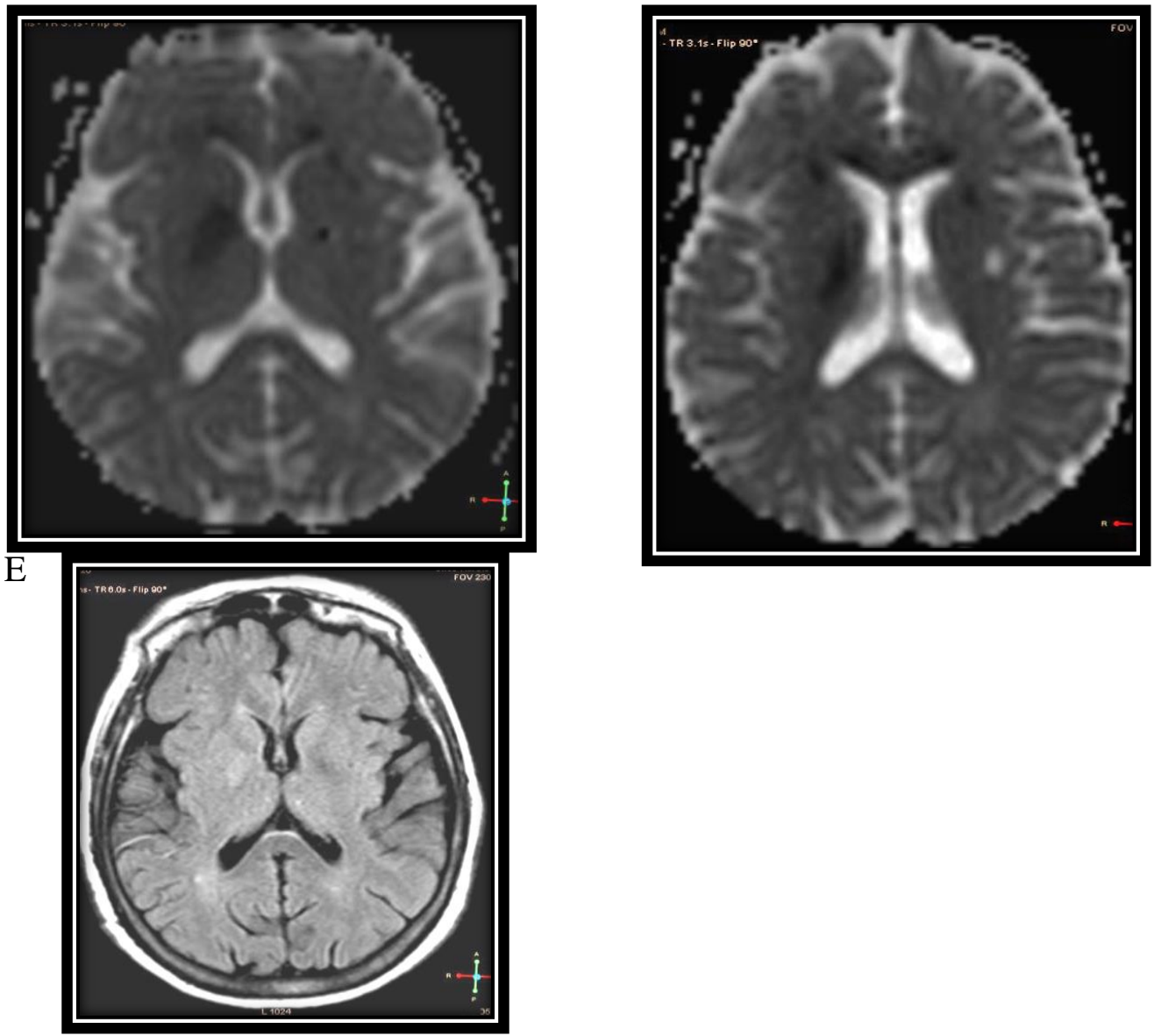

Fig. 2 (a-e): 41 - year- old woman with seizers and disturbed conscious level due to eclampsia (patient No.6 in table 1) Fig.(a,b): Axial DWI show Rt basal ganglia and Lt frontal abnormal high signal intensity of restricted diffusion on DWI and displays low signal intensity on their corresponding ADC map Fig.(c,d) suggestive of cytotoxic edema. Follow up FLAIR images. Fig.(e): Shows small subtle residual abnormal high signal in the Rt basal ganglia confirming ischemic insult and infarction. 
A

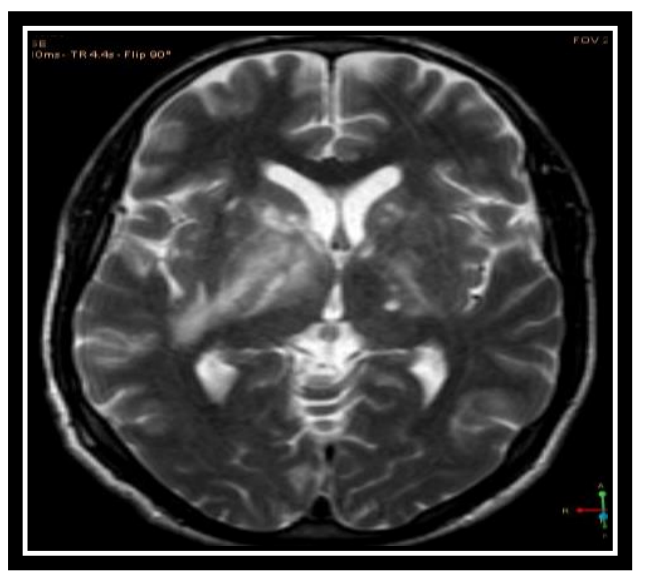

$\mathrm{C}$
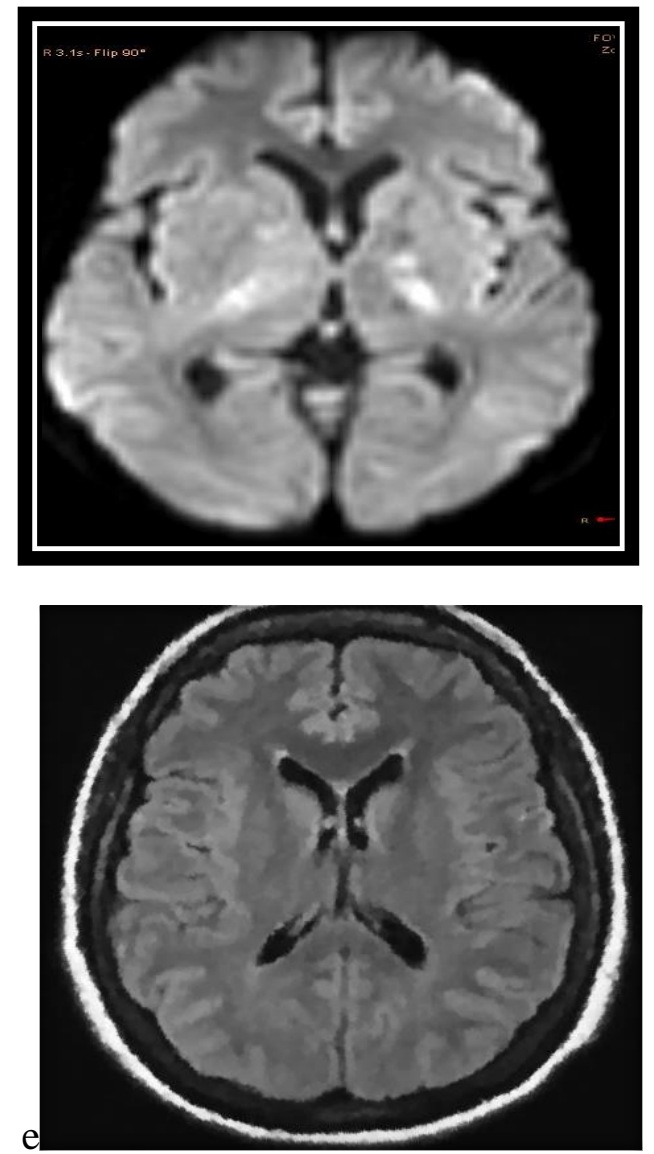

B

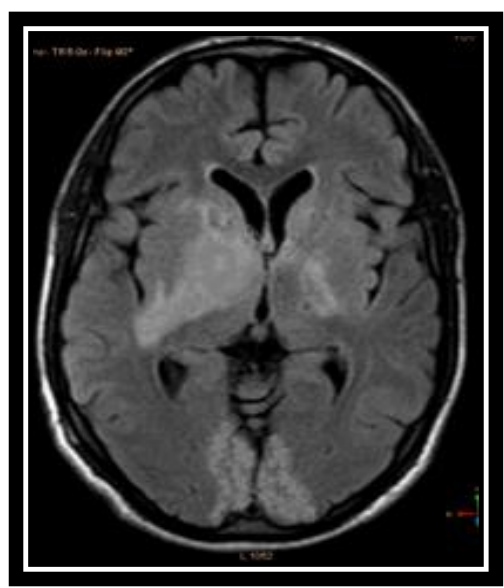

$\mathrm{D}$

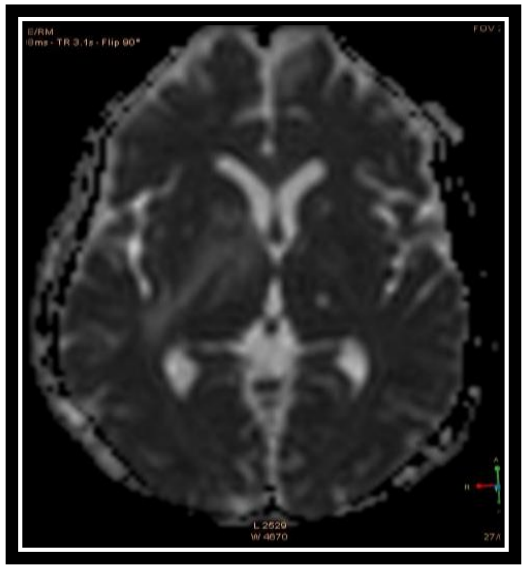

Fig. 3 (a-e): Antepartum eclampsia showing reversible lesions due to vasogenic edema in 38 years pregnant lady presented with hypertension, headache, visual disturbance \& seizures (patient no.8 in table 1). A,b,c,d were obtained 24 hours after the onset of symptoms. Fig. (a\&b): Axial T2WI \& FLAIR images show bilateral nearly symmetrical cortical and sub cortical high intense areas in both occipital lobes and both basal ganglia. Fig. (c\&d):DWI and ADC map showed increased signal with subsequent increased SI in ADC map (suggestive of vasogenic edema with T2 shin through phenomena). 6 weeks after delivery the hyper intense areas in initial FLAIR image disappear on follow up FLAIR image (Fig. e) denoting reversible lesions due to vasogenic edema. 
A

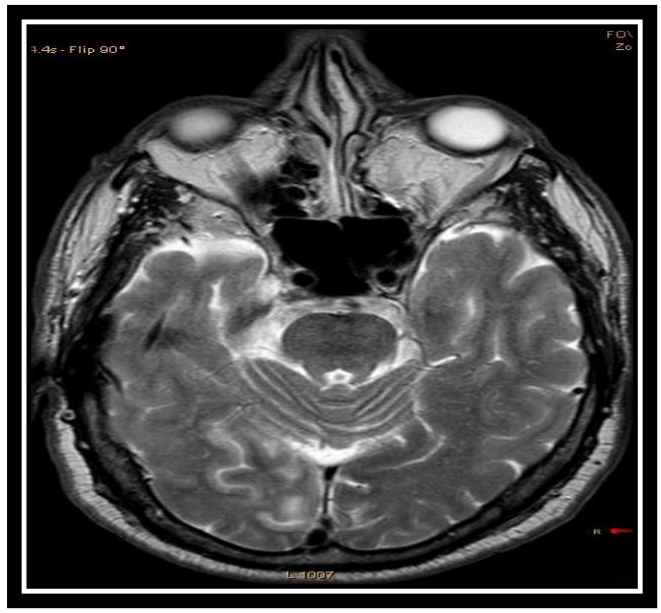

$\mathrm{C}$

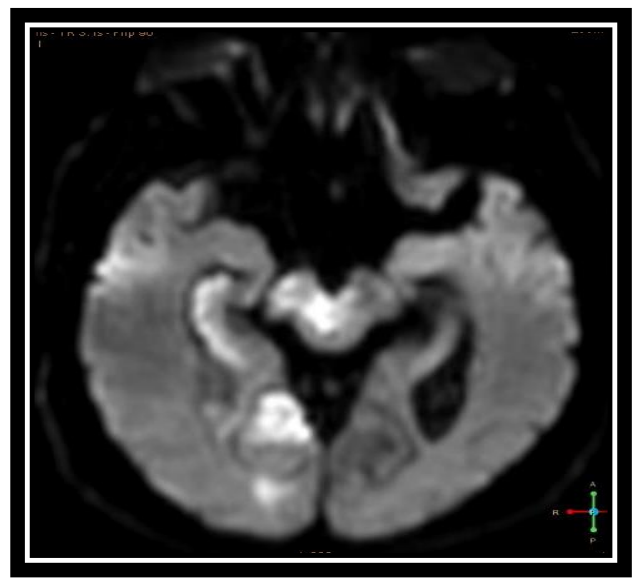

$\mathrm{E}$

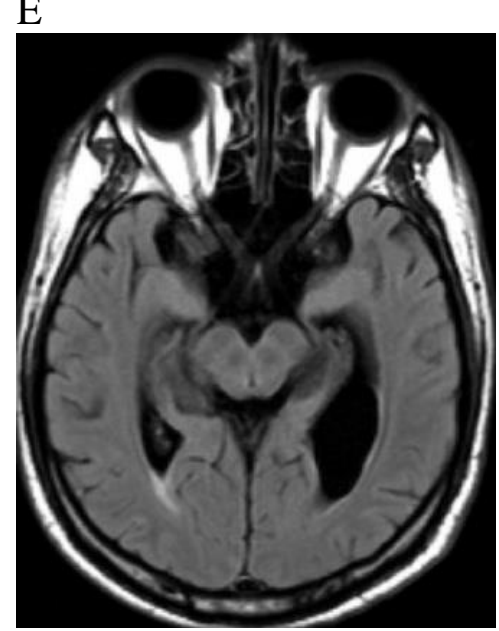

B

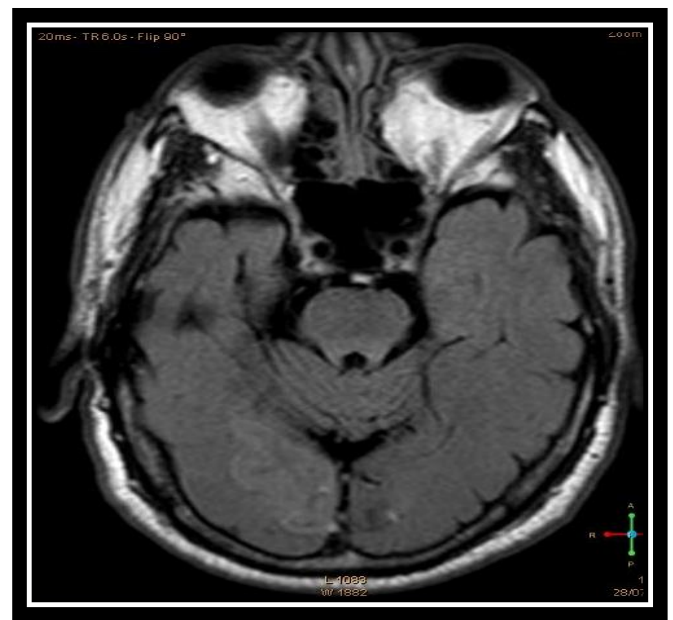

d

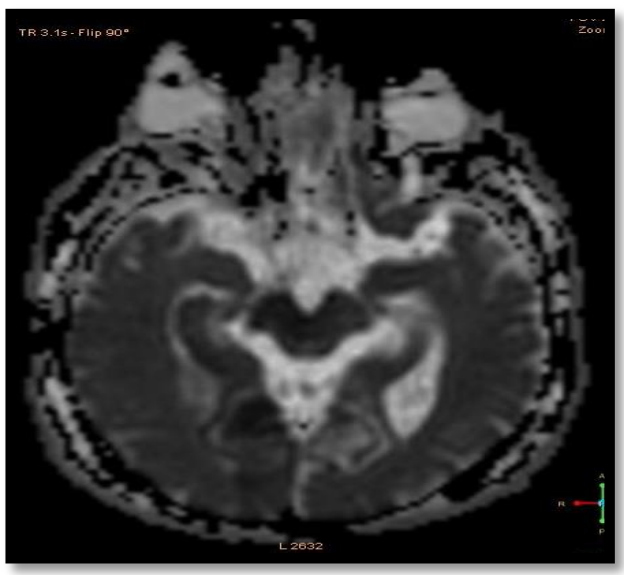

Fig. 4 (a-e): 45 - year- old woman with transient cortical blindness and hemiparesis due to eclampsia (patient No.9 in table 1). Fig. (a\&b): Axial T2WI and FLAIRImages show subtle high signal in cortical and sub cortical occipital lobes.Fig. (c\&d): DWI showed high signal with subsequent low SI in ADC map (suggestive of cytotoxic edema) involving occipital lobes, Rt temporal lobe, as well as brain stem (medulla) Fig. (e): Follow-up FLAIR image confirms the presence of infarction. 


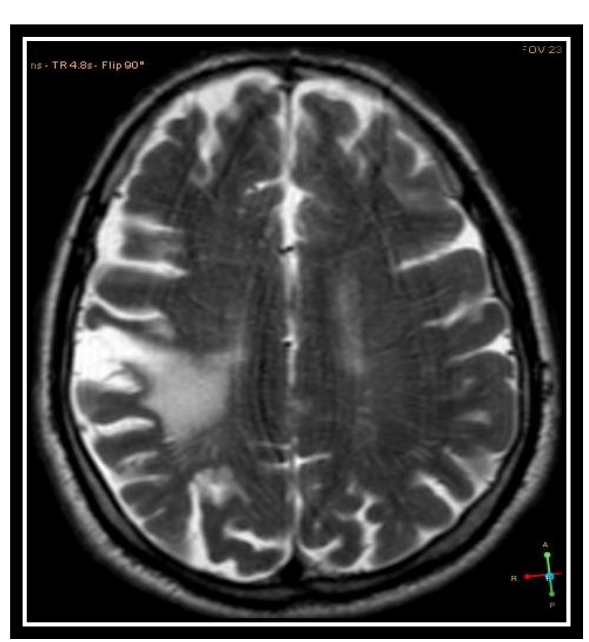

A

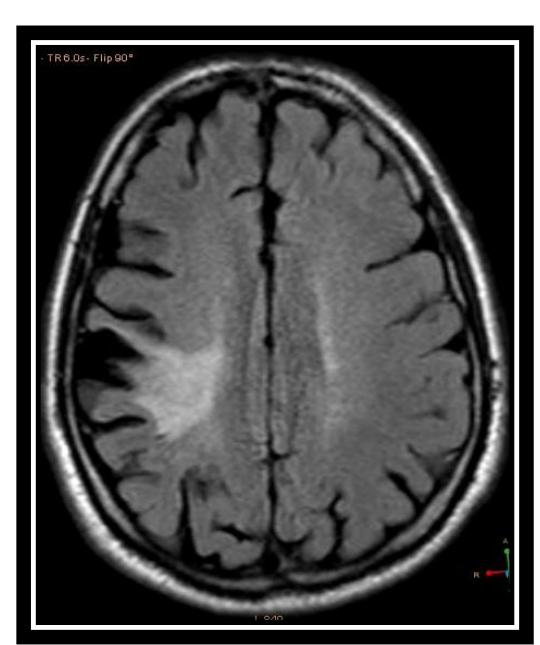

$\mathrm{b}$
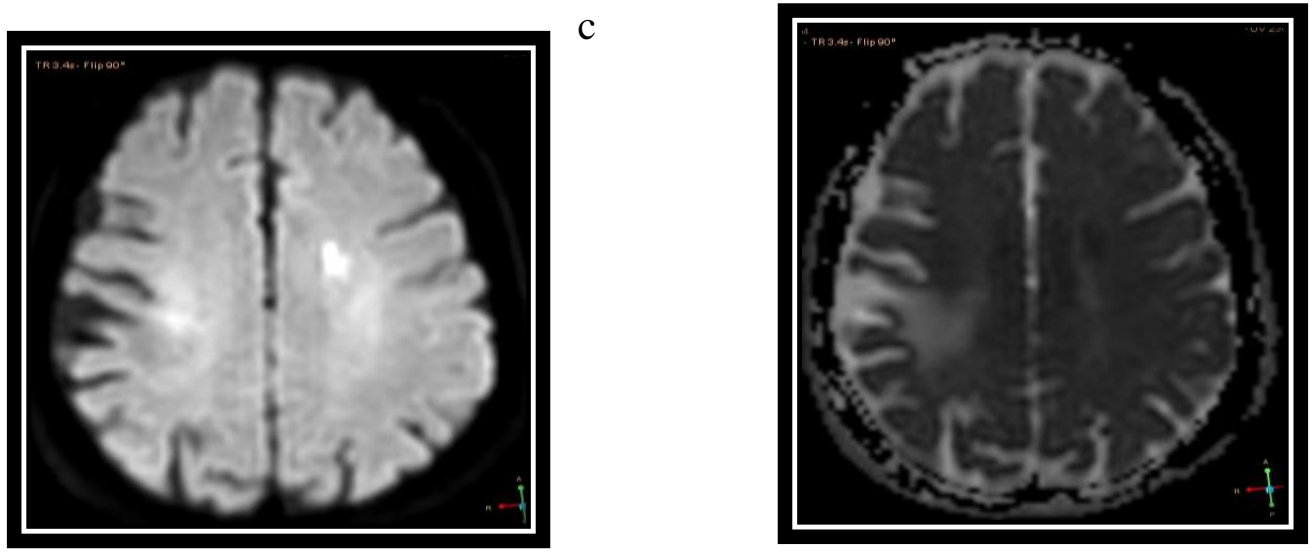

d

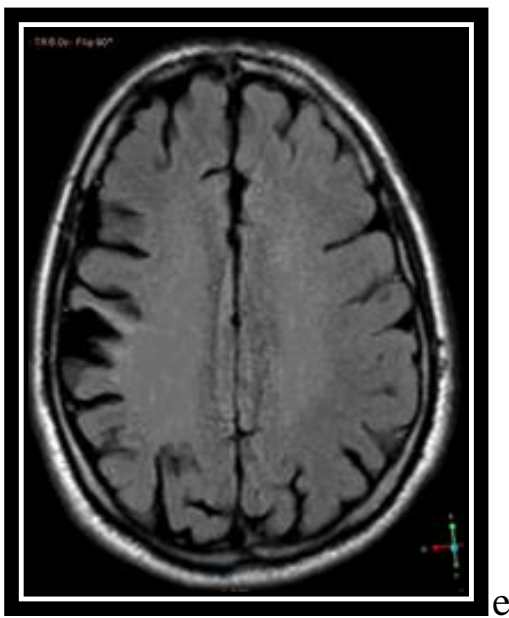

Fig. 5 (a-e): Antepartum eclampsia showing irreversible lesions (due to cytotoxic edema) \& reversible lesions (due to vasogenic edema) in a third trimester pregnant lady presented with severe hypertension, headache, seizures \& altered mental status (patient no. 15 in table 1). A,b,c,d were obtained 24 hours after the onset of symptoms and Fig. (e)were obtained 7weeks after delivery. Fig. (a\&b):Axial T2WI and FLAIR of the brain showed hyper intense areas in both parietal regions more in the right.Fig. (c\&d): Axial DWI and ADC map image of brain at same level show mainly hyperintense signal on DWI and ADC map (vasogenic edema and T2 shin through) as well as small hyperintense foci on DWIs that displayed hypointense signal on corresponding ADC map denoting infarction due to cytotoxic edema.Fig.(e): Follow-up FLAIR image showed complete resolution of the abnormal biparietal lesions with presence of small lacunar infarcts as subtle hyper intense foci in both parietal lobes. 


\section{3. RESULTS}

4. All patients presenting by signs and symptoms of pre-eclamptic and eclamptic encephalopathy, the most common presenting signs and symptoms in our patients were headache in 19 patients $(79.1 \%)$, seizures in 7 patients (29 \%), visual disturbances (blurred vision or hallucination) in 10 patients $(41.7 \%)$, disturbed level of consciousness (DLC) in 6 patient (25 $\%)$ and hemiparesis in 2 patient $(8.3 \%)$. Six patients were primigravida and 18 were multigravida. All patients were acutely hypertensive at their eclamptic episodes (with no previous history of chronic hypertension or renal disease). All patients' neurological examination was generally preceded by a rapid increase in blood pressure. For each patient, the blood pressure during the hypertensive crisis and the base line blood pressure obtained at least one week prior to the onset of neurologic symptoms are shown in table 1 .

5. All 24 patients had abnormal MR findings at initial scans manifested as multiple bilateral, nearly symmetrical hyperintense signals on T2 and FLAIR images involving the subcortical white matter and adjacent gray matter with posterior predominance. The lesions were more obvious and extensive on FLAIR images. The most common involved areas were: The occipital lobes in 22 patients $(91.6 \%)$, temporal lobes in 10 patients $(41.6 \%)$, parietal lobes in 8 patients $(33 \%)$, cerebellum in 2 patient $(8.3 \%)$, one case in brainstem $(4.1 \%)$ and in 5 patients $(20.8 \%)$ frontal lobes ware involved. Eleven patients (45.8\%) had basal ganglia involvement.

6. Comparing DWI and ADC maps in areas of increased T2 signal intensity showed that in the majority of patients (12 patients), DWI showed normal or decrease signal with subsequent increased; SI in ADC map (suggestive of vasogenic edema), and 8 patients showed high signal on DWI with also increased; SI in ADC map (suggestive T2 shin through and vasogenic edema) While 4 patients showed high signal intensity on DWI and low SI on ADC maps (suggestive of cytotoxic edema).

7. Follow up scanning was done after 6-8 weeks postpartum in all 24 patients. Complete resolution of the signal abnormalities in 20 patients who became clinically free (14 of them recovered completely within one week and remaining six within two weeks). The other four patients with persistent neurological deficit showed abnormal SI suggestive of cytotoxic edema on initial scans with persistent high signal abnormality on follow up scans by FLAIR images. 
Table 1: Clinical history, neurologic manifestation and blood pressure in 24 patients with preeclamptic and eclamptic encephalopathy.

\begin{tabular}{rrrrr}
\hline $\begin{array}{r}\text { Patients No } \\
\text { years }\end{array}$ & Clinical history & $\begin{array}{r}\text { Maximal blood } \\
\text { pressure }\end{array}$ & $\begin{array}{r}\text { Baseline blood } \\
\text { pressure }\end{array}$ \\
\hline 1 & 32 & Headache & $175 / 110$ & $120 / 82$ \\
\hline 2 & 31 & Blurred vision, DLC & $176 / 112$ & $125 / 85$ \\
\hline 3 & 30 & Headache & $150 / 100$ & $118 / 86$ \\
\hline 4 & 25 & Headache & $158 / 105$ & $115 / 80$ \\
\hline 5 & 37 & DLC\&seizures & $190 / 120$ & $105 / 75$ \\
\hline 6 & 41 & Headache & $165 / 106$ & $130 / 85$ \\
\hline 7 & 27 & Headache & $120 / 70$ \\
\hline 8 & 38 & Headache, visual hallucination \& seizures & $155 / 109$ & $130 / 75$ \\
\hline 9 & 45 & Blurred vision \&Hemiparessis & $190 / 117$ & $120 / 80$ \\
\hline 10 & 33 & Headache \& Seizures & $180 / 111$ & $125 / 85$ \\
\hline 11 & 40 & Headache \&visual hallucination. & $180 / 120$ & $135 / 85$ \\
\hline 12 & 39 & $150 / 110$ & $125 / 85$ \\
\hline 13 & 39 & DLC & $175 / 105$ & $120 / 75$ \\
\hline 14 & 28 & Headache \& visual hallucinations & $168 / 100$ & $115 / 75$ \\
\hline 15 & 40 & Headache \& seizures & $185 / 124$ & $105 / 30$ \\
\hline 16 & 26 & Headache & $174 / 107$ & $130 / 70$ \\
\hline 17 & 25 & Headache, seizures \&DLC & $130 / 70$ \\
\hline 18 & 44 & Headache, visual hallucination\& seizures & $150 / 90$ & $100 / 70$ \\
\hline 19 & 42 & Headache \& Blurred vision & $150 / 97$ & $110 / 70$ \\
\hline 20 & 42 & Headache \& Blurred vision & $154 / 101$ & $110 / 70$ \\
\hline 21 & 29 & Seizures \&Hemiparessis & $186 / 108$ & $120 / 80$ \\
\hline 22 & 45 & Headache \& DLC & $178 / 106$ & $115 / 75$ \\
\hline 23 & 38 & Headache \&DLC & $169 / 110$ & $125 / 85$ \\
\hline 24 & 39 & Headache \& visual hallucinations & $165 / 103$ & $115 / 80$ \\
\hline & & &
\end{tabular}


Table 2:Distribution, Signal intensity changes on DW and Its corresponding ADC encephalopathy and their outcome:

\begin{tabular}{|c|c|c|c|}
\hline $\begin{array}{r}\text { Patients } \\
\text { No }\end{array}$ & Lesion distribution & DWI signal & $\begin{array}{r}\mathrm{ADC} \\
\text { signal } \\
\end{array}$ \\
\hline 1 & Occipital & High & High Reversed(good) \\
\hline 2 & Occipital, frontal \& parietal & Low & High Reversed(good) \\
\hline 3 & Occipital, temporal, \& parietal & High & Low Residual(stroke) \\
\hline 4 & Occipital & Low & High Reversed(good) \\
\hline 5 & Occipital temporal \& basal ganglia & Iso & High Reversed(good) \\
\hline 6 & frontal\& basal ganglia & High & Low Residual(stroke) \\
\hline 7 & Occipital\& parietal & Iso & High Reversed(good) \\
\hline 8 & Occipital \& basal ganglia & High & High Reversed(good) \\
\hline 9 & Occipital, temporal \& brainstem & High & Low Residual(stroke) \\
\hline 10 & Occipital, Parietal, temporal & Low & High Reversed(good) \\
\hline 11 & Occipital, cerebellar \& basal ganglia & High & High Reversed(good) \\
\hline 12 & Occipital, temporal\& basal ganglia & Low & High Reversed(good) \\
\hline 13 & Occipital, frontal\& basal ganglia & High & High Reversed(good) \\
\hline 14 & Occipital\& basal ganglia\& parietal & Iso & High Reversed(good) \\
\hline 15 & $\begin{array}{r}\text { Parietal } \\
\end{array}$ & High & Low Residual(stroke) \\
\hline 16 & Occipital, temporal & Low & High Reversed(good) \\
\hline 17 & Occipital, temporal\& basal ganglia & Iso & High Reversed(good) \\
\hline 18 & Occipital, temporal\& basal ganglia & Iso & High Reversed(good) \\
\hline 19 & Occipital\& basal ganglia & High & High Reversed(good) \\
\hline 20 & Occipital, parietal & High & High Reversed(good) \\
\hline 21 & Occipital, temporal & Low & High Reversed(good) \\
\hline 22 & Occipital, frontal, parietal \& basal ganglia & Iso & High Reversed(good) \\
\hline 23 & Occipital, frontal \& cerebellar & High & High Reversed(good) \\
\hline 24 & Occipital, temporal & High & High Reversed(good) \\
\hline
\end{tabular}

\section{DISCUSSION}

Hypertension, edema, protinurea, and activation of coagulation cascade are the clinical presentations of pre-eclamptic \& eclamptic pregnant female or whose in the early puerperium, Untreated or severe forms of preclampsia can progress to eclampsia and manifested by convulsion. Manifestations at presentation time are not sufficiently specific to establish the diagnosis, so Imaging can confirm the diagnosis and can predict the course of brain edema in an acute phase in patients with eclamptic encephalopathy. Posterior reversible encephalopathy syndrome (PRES) is a heterogeneous group of disorders which share many clinical and radiographic features with hypertensive encephalopathy in which the patients experience headache, seizures and neurological disturbance most commonly visuals changes $(13,14)$.

Usual MR imaging mainly T2-weighted and FLAIR images helps in confirming the diagnosis by showing characteristic changes that include subcortical white matter hyperintense lesions predominantly posterior in distribution commonly the occipital lobes, often with involvement of the posterior temporal, parietal(15). Other deep white matter structures, such as, basal ganglia, and the white matter of the frontal or temporal lobes can also be 
affected(16,2).The most important MRI features are the reversibility of the condition in most of the cases hence the term Posterior reversible encephalopathy syndrome (PRES)(17,18).

The pathogenesis of eclampsia is debated. One hypothesis attributed the cause of ischemia \& cytotoxic edema to the vasospasm within the medium sized and large arteries supplying the posterior brain regions, and if ischemia is not successfully treated, it can proceed to frank infarction (19). According to another hypothesis, PRES lesions are primarily results from an acute increase in blood pressure that overcomes the myogenic vasoconstriction of cerebral arteries and arterioles leading to loss of autoregulatory capacity, BBB disruption, and vasogenic edema (20)

Current evidence indicated that both mechanisms could be, considering the autoregulation now as the underlying common pathogenesis mechanism(21).

In a healthy innervated subject, cerebral auto regulatory mechanism has both myogenic and neurogenic components that maintain constant brain perfusions. In patients with eclamptic encephalopathy the myogenic response is blunted by either passive overdistention of the vessel due to elevation in blood pressure or direct toxic effect on the endothelium. So the predilection of the posterior circulation territories is generally known to result from relatively sparsely sympathetic innervation of the vertebrobasilarcirculation(22).

The most common affected site was the parieto-occipital region which was present in $18(81.82 \%)$ (23). In our series we also confirmed the predilection for posterior circulation territories as The occipital lobes were involved in 22 patients $(91.6 \%)$ and the parietal lobes in 8 patients (33\%), but anterior circulation structures not excluded. Frontal lobe involvement was seen in $20.8 \%$ of our patients and was seen often obvious. These findings agree with Covarrubias et al.(7) who reported that in severe cases of eclampticc encephalopathy, the more richly innervated territories of the anterior circulation become susceptible to reversible encephalopathy.

Mueller-Mang et al., 2009 reported that most of the hypertensive patients with basal ganglia affection suffered from preeclampsiaeclampsia $(60 \%)$. Other studies, has reported the predilection for basal ganglia involvement in patients with preeclampsia-eclampsia (24). Another study including 18 patients with preeclampsia-eclampsia PRES, basal ganglia involvement has been detected in 10/18 $(55.5 \%)$ patients(24). This agrees with our study as eleven patients $(45.8 \%)$ had basal ganglia involvement.

The pathogenesis of basal ganglia involvement in preeclampsia-eclampsia PRES patients remains unclear. Rappaport et al (1990) reported that endothelial damage in patients with preeclampsia-eclampsia is associated with increase in circulating endothelial toxins, as well as antibodies against the endothelium (25)

In another study of PRES in eclamptic patients reported three patients(13.6\%), with a completely unilateral basal ganglia involvement (26).

The other regions of the cerebral hemispheres affected were the midbrain (9.09\%), basal ganglia (18.18\%), cerebellum(18.18\%), and thalamus in $(9.09 \%)$ (23). In our study temporal lobes were involved in 10 patients $(41.6 \%)$, cerebellum in 2 patient $(8.3 \%)$, and one case in brainstem (4.1\%).

Amin etal 2012,(26) found that most common clinical symptoms were seizures and altered mental status in approximately half of the patients, who included in their study, also Fugate et al., 2010 found that the most common clinical presentations were new-onset seizures, encephalopathy, headache, and visual disturbances. (27)

These did not differ from our study as we found the most common presenting signs and symptoms were headache $(79.1 \%)$, and visual disturbances $(41.7 \%)$, however, other manifestations as seizures (29\%), disturbed 
level of consciousness (DLC) $(25 \%)$ and hemiparesis in $(8.3 \%)$ were recorded.

Conventional MRI cannot clearly distinguish between different types of brain edema although vasogenic edema is predominantly involving the white matter while cytotoxic edema is involving the gray matter, white matter, or both yet tell now. Echoplanar diffusion weighted imaging (DWI) can clearly distinguish between the two. Regions with cytotoxic edema display hyperintense signal on DWI due to reduction in proton diffusion. However, vasogenic edema is characterized by increased extra cellular fluid with enhanced water diffusion and this may be seen as isointense or reduced signal brightness on DWI.

Vasogenic edema, however, may display hyper intense signal on DWI, named (T2-shine through). Thus, to discriminate if DWI hyper intensity is due to restricted diffusion (cytotoxic edema) or T2-shine, we have to estimate the underlying $\mathrm{ADC}$ in the tissue in question, as the ADC calculation is independent of $\mathrm{T} 2$ effects. A decreased ADC in the areas corresponds to hyper intense lesions on DWI represents restricted diffusion (cytotoxic edema). However, elevated ADC represents increased diffusion motion of water molecules, and thus vasogenicedema(12).

In diffusion study hyperintensity in DWI with hyperintensity in ADC map in 15 patients $(68.18 \%)$ was explained to be due to vasogenic edema (T2 shine through phenomenon and not due to true restriction of diffusion),.Noha Mohamed (2014). In this study, Most of patients (20 of 24 cases) demonstrated increased-diffusion in the areas of T2 hyper intensity consistent with vasogenic edema. These findings were further confirmed by complete resolution of the abnormal SI on follow up scans after lowering blood pressure by antihypertsive drugs. These results agree with many authors $(28,29,30)$. Not all areas of high signal on DWI were cytotoxic edema and this can be due to the T2-shine through effect ( seen in 8 patients $33.3 \%$ )and thus the correlation between ADC maps and DWI is very important to solve this diagnostic problem(they displayed low also high signal of unrestricted diffusion) (Fig. 1). This finding agrees with Cunningham et al.(12).

Noha Mohamed (2014) found that lesions in 4 patients $(18.1 \%)$ displayed hyperintensity in DWI with hypointensity in ADC map denoting the presence of cytotoxic edema and development of ischemic infarction with irreversible tissue damage as a common complication of PRES(23). In our study four patients showed restricted diffusion in the areas of T2-hyper intensity suggesting underlying cytotoxic edema, these findings were confirmed radiologically at follow up scan which showed persistent signal abnormality with persistent neurological deficits.These results agree with many results $(4,31,32)$.

\section{CONCLUSION}

There is typical radiologic involvement of the brain regions in eclampsia. The use of diffusion-weighted imaging with ADC allows an earlier and clearer regional detection, as well as, differentiation between cytotoxic and vasogenic edema, which can predict the development of infarction.

Our findings indicate that; in preeclamptic/ eclamptic patients, brain edema is mostly vasogenic (83.3\%), although ischemic/cytotoxic edema was observed less commonly ( $16.6 \%$ of our patients). This has important therapeutic and prognostic implications because the reversibility of the clinical and radiologic abnormalities is contingent on the prompt control of blood pressure, to avoid progression to irreversible cytotoxic edema and infarction.

\section{REFERENCES}

[1] Kaplan PW: Neurologic aspects of eclampsia. NeurolClin (2004) 22: 841-861.

[2] Fugate JE, Claassen DO, Cloft HJ, Kallmes DF, Kozak OS, Rabinstein AA. Posterior reversible encephalopathy syndrome:associated clinical and radiologic findings. Mayo ClinProc 2010;85(5):427-32

[3]Miza A. Posterior reversible encephalopathy a variant of hypertensive encephalopathy. J ClinNeurosci 2006:590-5. 
[4] Coppage KH and Sibai BM: Diagnosis and management of women with stroke during pregnancy/postpartum. Clinperinatol (2004) 31:853-868.

[5]Engelter ST, Provenzale JM and Petrella JR: Assessment of vasogenic edema in eclampsia using diffusion imaging. Neuroradiology (2000) 42:818-820.

[6] Shaeffer PW, Buonanno FS, Gonzalez RG et al.: Diffusion weighted imaging discriminate between cytotoxic and vasogenic edema in patients with eclampsia. Stroke (1997) 28:1082-1085.

[7] Covarrubias DJ, Lutemer PH and Campeau NG: posterior reversible encephalopathy syndrome: prognostic utility of quantitative diffusion weighted MR imaging, AJNR (2002) 33: (6): 1038-1048.

[8]Naqi R, Ahsan H, Azeemuddin M. Posterior reversible encephalopathysynd-rome: a case series in patients with Eclampsia. J PakMedAssoc 2010;60:394-7.

[9] Casey SO, Sampaio RC, Michel E et al: Posterior reversible encephalopathy syndrome: utility of FLAIR imaging in thedetection of cortical and subcortical lesions. AJNR (2000) 21:1199-1206.

[10] Aye H, Buonanno FS, Schaffer PW et al: posterior leukoenephaloptahy without severe hypertension, utility of Diffusion weighted MRI. Neurology (1998) 51:1369-1376.

[11] American. College of Obstetricians and Gynecologists.Diagnosis and. management of Preeclampsia and eclampsia.ACOG Practice Bulletin Obstetrics and Gynecology, (2002) 33. 99(1): 159.-167.

[12] Schwartz RB, Feske SK, Polak JF et al. 1998: Pre eclampsia- eclampsia. :-clinical and neuroradiological findings correlates and insights into the pathogenesis of hypertensive encephalopathy radiology (2002), 217:317379.

[13] Shah AK, Whitty JE: Brain MRI in peripartum seizures: usefulness of combined T2 and diffusion weighted MR imaging. J NeurolSci (1999) Jul 1; 166(2): 122-125

[14] Cunningham FG, Twickler DM, Fleckenstein JL et al: Cerebral infarction in eclampsia. American Journal of Obstetrics and Gynecology (2004) 190, 714-720
[15] Zak IT, Dulai HS, Kish KK. Imaging of neurologic disorders associated with pregnancy and the postpartum period. Radio-Graphics 2007;27:95-108.

[16] McKinney AM, Short J, TruwitCh L, McKinney ZJ, Kozak OS, SantaCruz KS, et al. Posterior reversible encephalopathy syndrome: incidence of atypical regions of involvement and imaging findings. AJR 2007;189:904-12.

[17]Kassem H, Provenzale JM, Petrella JR etal, Early diffusion weighted MR imaging abnormalities in sustained seizures activity. AJR (2000); 174:1304-1306.

[18]Manfredi M, Beltramello A, Bongioranni LG et al., Eclamptic encephalopathy: imaging and pathogenetic considerations. ActaNeurolScand (1997); 96:277-282.

[19] Koch S, Rabinstein A, Falcone $S$ et al.: Diffusion weighted imaging shows cytotoxic and vasogenic edema in eclampsia. AJNR( 2001);22:1068-1070.

[20]Marilyn J. Cerebral circulation in pregnancy and eclampsia.Hypertens Am Heart Assoc 2007;50:14-24.

[21] Port J and Beauchamp N: reversible intracerbral pathologic entities mediated by vascular auto regulatory dysfunction. Radiographics(1998) ; 18: 353-367.

[22] Provezlae JM, Peterella JR and CRUZ JR etal, quantitative assessment of diffusion abnormalities in posterior reversible encephalopathy syndrome. AJNR (2001); 22 :( 8): 1455-1461.

[23]Noha Mohamed, AbdelMaboud Ibrahim, Manal E. Badawy. MRI imaging of posterior reversibleencephalopathy syndrome associated with pregnancy.The Egyptian Journal of Radiology and Nuclear Medicine (2014) 45, 505-510.

[24] Mueller-Mang C, Mang T, Pirker A, Klein K, Prchla C, Prayer D. Posterior reversible encephalopathy syndrome: do predisposing risk factors make a difference in MRI appearance? Neuroradiology 2009;51(6):373-83

[25] Rappaport VJ, Hirata G, Yap HK, Jordan SC. Anti-vascular endothelial cell antibodies in severe preeclampsia. Am J ObstetGynecol 1990;162:138-46.

[26] Amin MF, GAWAD EA, EL Ameen NF. Posterior reversible encephalopathy syndrome 
with atypical regions in eclamptic patients: A challenge for radiologists. The Egyptian Journal of Radiology and Nuclear Medicine (2012) 43, 595-601

[27] Fugate JE, Claassen DO, Cloft HJ, Kallmes DF, Kozak OS, Rabinstein AA. Posterior reversible encephalopathy syndrome: associated clinical and radiologic findings. Mayo ClinProc 2010;85(5):427-32

[28] Zeeman GG, Fleckenstein JL, Twickler DM: Cerebral infarction in eclampsia. Am J ObstetGynecol (2004); 190(3): 714-20

[29]Mulkern RV, Gudbjartscan H et al. Diffusionweighted MR imaging in hypertensive encephalopathy: clues to pathogeneses. AJNR (1998); 19:859-866.
[30] Watanabe Y, Mitoma M, Toxuda Y et al :Eclamptic Encephalopathy : MRI, including diffusion-weighted images. Neuroradiology (2002); 44:981-985.

[31]Bartynski. W, Z. Zeigler, R. Shadduck, R. K. etal. Neuroimaging in Posterior Reversible Encephalopathy Syndrome J Neuroimaging, (2004); 14(2): 89 - 96.

[32] Matsumoto K, Loch, Pierce AR et al; Role of vasogenic edema and tissue cavitations in ischemic evolution of diffusion-weighted imaging: comparison with multiparameter MR and immunohistochemistry. AJNR(1995);16:11071115. 